Received: 2012.05.17

Accepted: 2012.05 .24

\section{Clinical and radiological features of nonfamilial cherubism: A case report}

\author{
Justyna Wagel ${ }^{1}$, Klaudiusz kuczak², Barbara Hendrich ${ }^{1}$, Maciej Guziński ${ }^{1}$, \\ Marek Sąsiadek ${ }^{1}$
}

1 Department of General and Interventional Radiology and Neuroradiology, Wrocław Medical University, Wrocław, Poland 2 Department and Clinic of Maxillo-Facial Surgery, Wrocław Medical University, Wrocław, Poland

Author's address: Justyna Wagel, Department of General Radiology, Interventional Radiology and Neuroradiology, Wrocław Medical University, Borowska 213 St., 50-556 Wrocław, Poland, e-mail: jwagel@gmail.com

\section{Summary}

Background: Cherubism is an uncommon hereditary benign fibro-osseous disorder characterized by bilateral enlargement of the mandible and the maxilla that presents with varying degrees of involvement and a tendency toward spontaneous remission. On radiography cherubic lesions appear as cystic multilocular radiolucencies limited to the jaw bones.

Case Report: $\quad$ A 5-year-old boy was referred to the Department of Maxillo-Facial Surgery due to deformation of the lower and middle section of the face and displacement or absence of teeth. A panoramic radiograph and a computed tomography revealed extensive multilocular, bilateral radiolucent areas and marked bony expansion in the mandible and maxilla, with sparing of the mandibular condyles. Histopathological evaluation of an incisional biopsy of the left maxilla and genotypic characterization confirmed the diagnosis of cherubism.

Conclusions: $\quad$ The radiologic characteristics of cherubism are not pathognomonic but the diagnosis is strongly suggested by bilateral relatively symmetric jaw involvement that is limited to the jaw bones and, together with clinical and histopathologic findings, enables the diagnosis of cherubism. Genotypic characterization confirms the diagnosis.

Key words: $\quad$ cherubism $\bullet$ mandible $\bullet$ maxilla $\bullet$ CT $\bullet$ panoramic radiograph

PDF file: $\quad$ http://www.polradiol.com/fulltxt.php?ICID $=883375$

\section{Background}

Cherubism was first described in 1933 by William Jones, a Canadian radiologist, as a familial multilocular cystic disease of the jaws with accompanying swelling of the cheeks [1]. Cherubism is a rare non-neoplastic, self-limiting fibro-osseous disorder characterized by bilateral cystic expansion of the mandible or/and maxilla in the childhood. Affected children appear normal at birth and have normal mental development. Bone expansion becomes noticeable in early childhood and grows progressively until puberty, then the lesions usually gradually resolve. The lesions are painless and more or less symmetrical. Frequently, cherubism is accompanied by dental arch and dental eruption abnormalities [2-7]. Extragnathic skeletal involvement is rarely observed. The rounded face, resulting from jaw hypertrophy and upward gaze with exposure of the sclerae below the pupil caused by elevated orbital floor, gives the patient appearance reminding the cherubs depicted in Renaissance paintings. Lesion regression is expected to occur spontaneously by the end of adolescence, resolving by middle age $[3,4]$.

Radiographically, lesions appear as cystic multilocular radiolucencies, often beginning near the mandibular angle and spreading to the mandibular ramus and body. Maxillary lesions may occur at the same time. Frequently, ectopic unerupted teeth are involved by lesions [4].

In this study, we present a case of a child with nonfamilial form of cherubism and discuss the clinical and radiologic characteristics of this condition. 


\section{Case Report}

A 5-year-old boy was referred to the Department of MaxilloFacial Surgery because of deformation of the lower and middle section of the face. The boy presented with a painless symmetrical progressive swelling of the cheeks (Figure 1) and bilateral submandibular lymphadenopathy. Intraoral clinical examination disclosed widening of the alveolar processes and displacement or absence of teeth. A panoramic radiograph revealed extensive bone destruction involving the mandibular body, angles and both ascending rami, and the maxilla with multilocular radiolucent areas and marked bony expansion. The lesions spared the mandibular condyles, but concerned the alveolar processes and the maxillary tuberosities. Many of the upper and lower teeth had been lost. The lesions were accompanied by displacement and impaction of erupted teeth and dental follicles (Figure 2).

Unenhanced computed tomography (CT) with three-dimensional reconstruction clearly revealed destruction of the maxilla and mandible with soap bubble-like bone remodeling, sparing of the mandibular condyles, and thinning of the adjacent cortical rims (Figure 3). The partially overgrown maxilla expanded toward the orbits, to a greater extent on the right side. Front wall of the maxilla was also expanded by intraosseus changes. The maxillary sinuses were surrounded by modified tissue, yet with aerial space. The body of the maxilla presented bilateral overgrowth. These symptoms are typical of cherubism. On the base of the family history, it might be presumed that this was probably a non-familial case of the disease because there was no other member of the family with a similar condition, such as an autosomal dominant inherited genetic disease. Laboratory tests, which included serum calcium and phosphorus levels, were normal. Histopathological evaluation of an incisional biopsy of the left maxilla showed giant-cell, dysplastic cystic bone tissue. The diagnosis of cherubism was confirmed by genotypic characterization (peripheral blood lymphocyte DNA was screened for mutations in the SH3BP2 gene). Because of the self-limiting nature of the disease, active phase of the disease and absence of any disturbances, there was no need to perform any surgery and the patient was scheduled for regular follow-up for 20 years.

At age 7.5, due to the progression of intraosseus giant-cell lesions of the jawbones, calcitonin therapy was introduced for a period of 6-12 or 24 months, depending on the therapeutic effect.

\section{Discussion}

Cherubism is a rare, painless, self-limiting osseous disorder in children and adolescents. The radiologic characteristics of cherubism are not pathognomonic but the diagnosis is strongly suggested by bilateral relatively symmetric jaw involvement that is limited to the maxilla and mandible. Imaging typically shows expansive remodeling of the involved bones, thinning of the cortices, multilocular radiolucencies with a coarse trabecular pattern and absence of periosteal reaction $[2,3]$.

This is a genetic disease in which the trait is transmitted in an autosomal dominant pattern with a marked variation in clinical expression. The penetrance may be up to $100 \%$ in men and $50 \%$ to $70 \%$ in women $[3,4,6,8]$. The condition was initially characterized as exclusively familial, however, sporadic cases have been also reported. The latter may result from incomplete penetrance or new mutations $[2-4,6,8]$.

Cherubism is known to be related to the mutations in the gene encoding the binding protein SH3BP2 on chromosome 4 p16.3 $[3,4,8]$. SH3BP2 gene mutations cause dysregulation of the Msx-l gene which is involved in the regulation of mesenchymal interaction in craniofacial morphogenesis [4].

Affected children appear normal at birth. Bilateral painless swelling tends to begin within a range of 14 months to 12 years of age, usually within the first several years of life. The lesions characteristically continue a pattern of variable enlargement of the jaws until puberty and does not progress later. During the late teens, the disease may undergo spontaneous, gradual, partial or complete involution, leading to remineralization of the lesions and sclerotic remodelling in adulthood. Maxillary lesions are the first to regress, while mandibular lesions are often still active at the age of 20. Facial appearance may return to almost normal by the fourth or fifth decade of life. However, some patients request surgical recontouring of their residual deformity during their twenties $[2-4,8,9]$.

Radiographically, cherubism is characterized by bilateral, well-defined, multilocular radiolucent areas within the jawbones. The lesions usually originate in the area of the mandibular angle and spread to the ascending rami and body of the mandible. Maxillary involvement is less frequent and usually less extensive, but always accompanies mandibular involvement. The extent of the lesions varies from minor to massive involvement of both jaws $[4,8,9]$. This may occasionally be associated with other extragnathic cystic foci $[3,4,6]$. The radiographic and CT signs of cherubism are: expansive bone remodeling, cortex thinning, scalloping and disruption; multilocular radiolucencies with a coarse trabecular pattern and soft-tissue density within, dental anomalies, absence of periosteal reaction, and sparing of the condyles $[2,10]$. The latter feature has been described as a pathognomonic of cherubism, however, a few cases of patients with condylar involvement have been reported in the literature. Therefore the finding of condylar involvement should not exclude the diagnosis of cherubism if other characteristic lesions are seen within the jawbones. CT scanning helps to provide a clear definition of the extent of disease $[2,9,11]$. This is difficult to establish on the base of plain CT, therefore multiplanar and three-dimensional reconstructions are obligatory [12]. Magnetic resonance imaging (MRI) features of cherubism described in the literature are nonspecific, but this examination is useful in elucidating the accurate extent of the cherubic lesions, particularly their relationship to the orbits and the optic nerves $[2,9,13]$. In our case, there were no indications to perform an MR examination.

Radiographically, cherubism is similar to fibrous dysplasia and has been previously described as a familial form of fibrous dysplasia localized to the jaw. However, genetic analysis has shown that the cherubism results from 

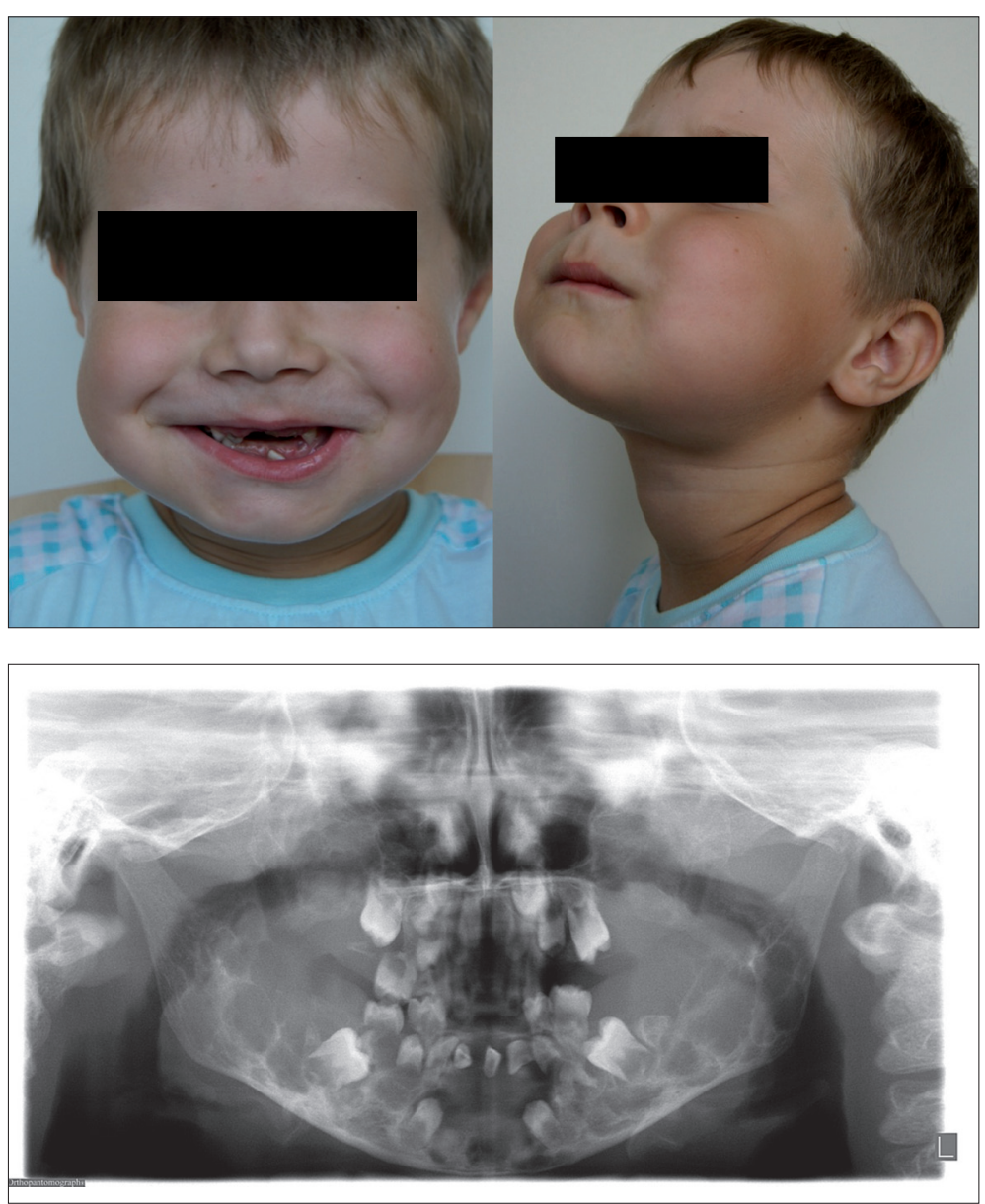

Figure 1. Patient presents enlargement of the lower and middle section of the face and characteristic swollen cheeks.
Figure 2. Panoramic radiograph shows expansive multiloculated cystic lesions distributed in the mandible and maxilla with loss and medial displacement of the deciduous teeth. different mutations than four subtypes of fibrous dysplasia (monostotic, polyostotic, craniofacial and McCune-Albright syndrome) and therefore is nowadays considered as a separate entity at the molecular level $[2,3,9,14]$.

The lesions in cherubism may cause abnormal patterns of teeth eruption, as well as teeth agenesis, premature loss of deciduous teeth and the presence of ectopic or retained teeth [4]. Widening of the alveolar ridges is common. Expansion of the maxillary alveolus sometimes resembles V-shaped palatal arch. In the cases where symmetry is lost, minor signs of the disease are useful, such as: gum epulis, enamel hypoplasia and gum overgrowth. Those minor signs can also be useful to diagnose cherubism in the relatives of affected children, in order to define a comprehensive family history. Cervical lymph nodes, which are usually involved during the active phase of the disease, are non-tender on palpation, mobile, large, and discrete $[3,5,6,10]$.

In some cases, maxillary expansion pushes the orbital base upward and causes upturned eyes and exposure of lower rim of the sclera. Significant ocular disturbances, such as lid retraction, proptosis, superior globe displacement and diplopia may occur [3,4]. Progression of the orbital involvement rarely may prolong beyond puberty, after stabilization or regression of the lesions in the jaws [5].

With adulthood, the cystic areas in the jaws become re-ossified giving irregular patchy sclerosis and a classical, but not specific, ground-glass appearance [6]. Sometimes sclerotic areas can replace radiolucent multilocular regions in the fifth decade of life [4].

In 1992, Marck and Kudryk proposed a grading system for cherubism depending on the location of the lesions. Accordingly, grade 1 concerns bilateral involvement of mandibular rami, grade 2 corresponds to the involvement of both mandibular rami and maxillary tuberosities, grade 3 includes massive involvement of the entire maxilla and mandible except for the condyles, while grade 4 relates to the involvement of the orbits with ocular disturbances as well as the grade 3 lesions [15]. In our patient, the lesions were classified as grade 3 , according to the grading system mentioned above.

Radiographic differential diagnosis of cherubism includes craniofacial fibrous dysplasia, brown tumor of hyperparathyroidism, Jaffe-Campanacci syndrome, familial gigantiform cementoma and odontogenic cyst. Although cherubism and craniofacial type of fibrous dysplasia show radiologic similarities, they may be distinguished clinically and histologically. Features more specific for the diagnosis of cherubism include bilateral mandibular involvement, limitation to the maxilla and mandible, and involution at the time of puberty. Brown tumor and Jaffe-Campanacci syndrome are distinguished on clinical grounds and are readily eliminated from the differential diagnosis on this base. Familial gigantiform cementoma is a rare osseous disorder 


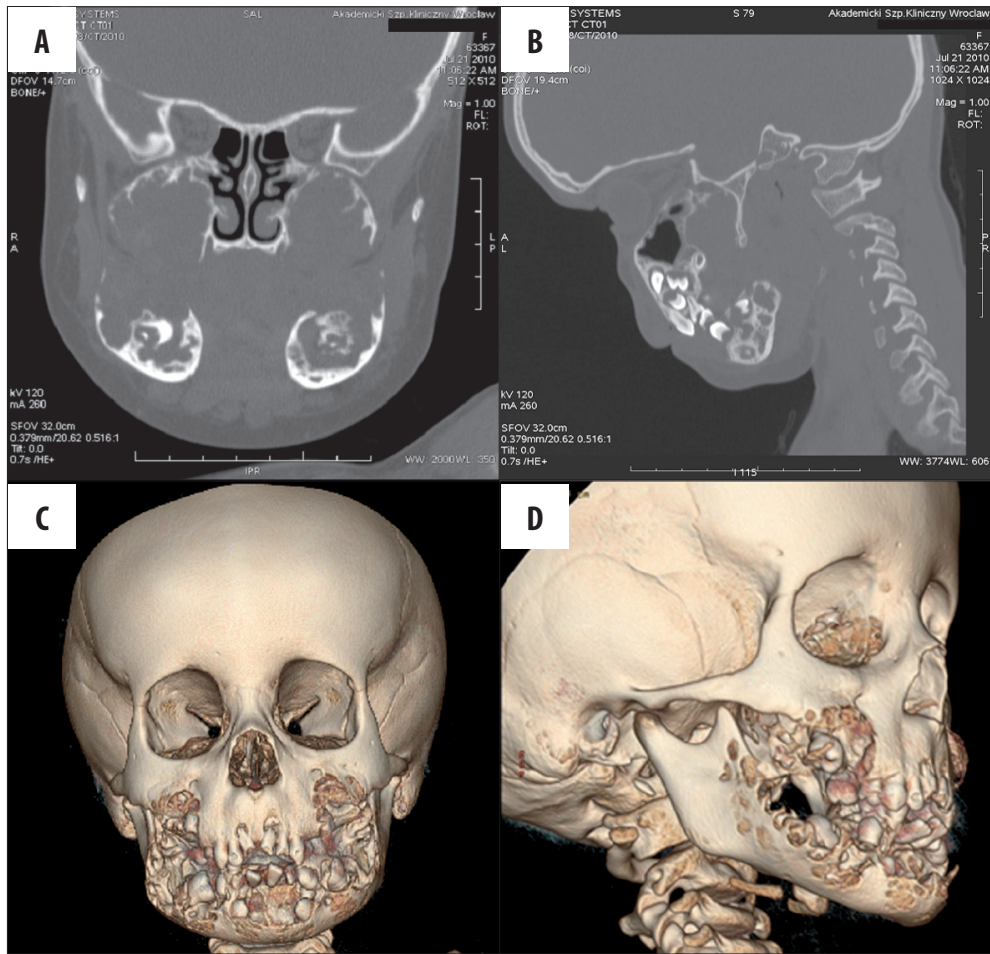

Figure 3. Coronal (A), sagittal (B) bone window CT and $3 \mathrm{D} C \mathrm{C}$ reconstruction $(\mathbf{C}, \mathbf{D})$ revealed destruction of the maxilla and mandible with soap bubble-like remodeling, cortical scalloping with sparing of the mandibular condyles.

characterized by the production of cementum in the lesions. Mandibular and maxillary involvement are often present, however, the lesions may extend into the orbits and nasal septum, locations that exclude the diagnosis of cherubism. Besides, gigantiform cementoma lesions are located primarily in the maxilla and are enlarged in a focal rather than diffuse pattern. Bilateral odontogenic cysts are rare in the first 5 years of life $[2,3,9,13]$.

Histological examination has limited value for diagnosis, showing abundant multinucleated giant cells scattered in a stroma of vascularized fibrous connective tissue [5]. These features are similar to the other giant cell-containing fibro-osseous diseases such as brown tumor of hyperparathyroidism, giant cell tumor, as well as central and peripheral giant-cell granuloma. As a result, the diagnosis also depends on clinical findings $[4,6,8]$. Central giant-cell granuloma may be excluded on clinical grounds because it presents itself slightly later (between 10 and 30 years of age), it is not a bilateral condition, is not inherited, does not regress in adulthood and usually involves predominantly the anterior mandible $[5,8]$. Giant-cell tumor recalls cherubism histopathologically, but it is unusual in the jaws, affects patients between the ages of 20 and 40 years and is seen unilaterally [5]. On the other hand, bone changes in hyperparathyroidism rarely affect the jaw in an isolated manner and produce abnormal serum calcium, phosphorus, and alkaline phosphatase levels [5,8]. Cherubism usually does not affect osseous metabolism; bone markers are generally at normal levels with respect to age [3-6]. In hyperparathyroidism, radiographic controls also reveal generalized bone loss [3]

As cherubism is generally a self-limiting condition and regresses with age, treatment depends on the clinical course of the disease and is indicated only in the cases of esthetic or functional problems. Most investigators prefer waiting until the end of puberty before performing a surgery. Surgery is indicated only in more aggressive cases characterized by functional impairment such as speech, chewing or swallowing, and ocular disturbances, or with the presence of major deformities that may cause psychological problems for the patient. Surgical removal of the fibrotic lesions does not modify the natural course of the disease and gives temporary relief, but may provoke rapid recurrence or exacerbate the disease if the patient is operated on during the active phase $[3,5,6,8,10]$.

Calcitonin therapy is known to cause regression in central giant-cell granuloma of the jaws by the inhibition of bone resorption by multi-nucleate cells (osteoclasts), which are also present in cherubic lesions, and, as an antiresorptive agent, seems to be effective in reducing cystic lesions in the jaw in patients with cherubism $[3,4,8,16]$.

Radiotherapy is contraindicated in cherubism due to the risk of osteoradionecrosis, induction of malignancy and growth disturbances $[3,5,6,10]$.

Cherubism may be associated with other genetic diseases, such as Ramon's syndrome, Noonan's syndrome (pseudo-Turner syndrome), neurofibromatosis type 1 , Jaffe-Campanacci syndrome and fragile $\mathrm{X}$ syndrome $[3,4,10,17-19]$

\section{Conclusions}

The radiologic characteristics of cherubism are not pathognomonic and the diagnosis of cherubism is based on a combination of clinical, radiographic, and histological findings. Histological examination has limited value for diagnosis. 


\section{References:}

1. Jones WA: Familial multilocular cystic disease of the jaws. Am J Cancer, 1933; 17: 946-50

2. Beaman FD, Bancroft LW, Peterson JJ et al: Imaging Characteristics of Cherubism, Am J Roentgenol, 2004; 182(4): 1051-54

3. Ozkan Y, Varol A, Turker $\mathrm{N}$ et al: Clinical and radiological evaluation of cherubism: a sporadic case report and review of the literature. Int J Pediatr Otorhinolaryngol, 2003; 67(9): 1005-12

4. Carvalho Silva E, Carvalho Silva GC, Vieira TC: Cherubism: Clinicoradiographic Features, Treatment, and Long-Term Follow-Up of 8 Cases. J Oral Maxillofac Surg, 2007; 65(3): 517-22

5. Colombo F, Cursiefen C, Neukam FW et al: Orbital Involvement in Cherubism. Ophtalmology, 2001; 108(10): 1884-88

6. Lannon DA, Earley MJ: Cherubism and its charlatans. Br J Plast Surg, 2001; 54(8): 708-11

7. Wang CN, Song YL, Peng B et al: The aggressive form of cherubism: Report of two cases in unrelated families. Br J Oral Maxillofac Surg, 2006; 44(4): 322-24

8. Peñarrocha M, Bonet J, Mínguez JM et al: Cherubism: A Clinical, Radiographic, and Histopathologic Comparison of 7 Cases. J Oral Maxillofac Surg, 2006; 64(6): 924-30

9. Jain V, Sharma R: Radiographic, CT and MRI features of cherubism Pediatr Radiol, 2006; 36(10): 1099-104

10. Roginsky VV, Ivanov AL, Ovtchinnikov IA et al: Familial cherubism: the experience of the Moscow Central Institute for Stomatology and Maxillo-Facial Surgery. Int J Oral Maxillofac Surg, 2009; 38(3): $218-23$
11. Bianchi SD, Boccardi A, Mela F et al: The computed tomographic appearances of cherubism. Skeletal Radiol 1987; 16: 6-10

12. Myga-Porosiło J, Skrzelewski S, SragaW et al: CT Imaging of facial trauma. Role of different types of reconstruction. Part I - bones. Pol J Radiol, 2011; 76(1): 41-51

13. Atalar $\mathrm{MH}$, Arslan $\mathrm{M}$, Aker $\mathrm{H}$ et al: Cherubism: an unusual cause of bilateral mandibular swellings. Eur J Radiol Extra, 2004; 49: 11-15

14. Mangion J, Edkins S, Goss AN et al: Familial craniofacial fibrous dysplasia: absence of linkage to GNASl and the gene for cherubism. J Med Genet, 2000; 37(11): E37

15. Marck PA, Kudryk WH: Cherubism. J Otolaryngol, 1992; 21: 84-87

16. de Lange J, van den Akker HP, Scholtemeijer M: Cherubism treated with calcitonin: report of a case. J Oral Maxillofac Surg, 2007; 65(8): 1665-67

17. Martínez-Tello FJ, Manjón-Luengo P, Martin-Pérez M et al: Cherubism associated with neurofibromatosis type 1 and multiple osteolityc lesions of both femurs; a perviously undescribed association of findings. Skeletal Radiol, 2005; 34(12): 793-98

18. Neumann TE, Allanson JE, Kavamura I et al: Multiple giant cell lesions in patients with Noonan syndrome and cardio-faciocutaneous syndrome. Eur J Hum Genet, 2009; 17(4): 420-25

19. Slezak R, Luczak K, Kalscheuer V et al: Noonan-like /Multiple Giant Cell Lesion Syndrome in two adult patients with SOS1 gene mutations. Clin Dysmorphol, 2010; 19(3): 157-60 